\title{
Immuno-oncology-the new paradigm of lung cancer treatment
}

\author{
D.E. Dawe $M D M S c,{ }^{*}$ C.H. Harlos $M D,{ }^{*}$ and R.A. Juergens $M D \mathrm{PhD}^{\dagger}$
}

\begin{abstract}
Systemic therapy is an essential part of treatment for all patients with small-cell lung cancer (SCLC) and for most patients with non-small-cell lung cancer (NSCLC). Standards of care have evolved dramatically since 2009, especially in the setting of incurable or advanced NSCLC. Part of that evolution has been the incorporation of immuno-oncology drugs, especially immune checkpoint inhibitors (ICIs) into multiple therapeutic scenarios.

In the present review, we discuss the role of the immune system in lung cancer and the previous failures of immunotherapy for patients with lung cancer. We then provide an overview of the existing evidence for the use of ICIs in patients with advanced NSCLC that is either treatment-naïve or pretreated, for consolidative treatment after chemoradiotherapy in stage III NSCLC, and for palliative therapy in patients with SCLC. Finally, we discuss duration of treatment, special populations, and the future of immuno-oncology for patients with lung cancer. Overall, we provide an evidence-based snapshot of immuno-oncology agents in the treatment of lung cancer up to early 2019.
\end{abstract}

Key Words Immuno-oncology, lung cancer, immunotherapy, PD-1, PD-L1

Curr Oncol. 2020 April:27(S2)78-86

www.current-oncology.com

\section{INTRODUCTION}

Lung cancer is the number one cause of cancer-related death both in Canada and worldwide. In Canada, 28,600 people were diagnosed with lung cancer in 2017, and 21,100 died. Worldwide, 2,093,876 people were diagnosed, and $1,761,007$ died $^{1,2}$. The number of lung cancers diagnosed in North America is driven by smoking rates and demographic shifts (growing populations and an increasing number of older adults).

Histologically, lung cancer is subdivided into nonsmall-cell lung cancer (NSCLC), which accounts for $85 \%$ of diagnosed lung cancers, and small-cell lung cancer (SCLC), which accounts for $15 \%^{3}$. Surgery is the mainstay of treatment for stages I and II NSCLC, but $75 \%$ of lung cancers are diagnosed at stage III or IV, when resection is no longer possible and the likelihood of mortality is high. Systemic therapy is an essential part of treatment for all patients with SCLC and for those with stage III or IV NSCLC. Standards of care (SOCs) have evolved dramatically since 2009, especially in the setting of incurable or advanced NSCLC $^{3}$.

In 2009, publication of the IPASS trial established the importance of molecular testing in patients with metastatic NSCLC $^{4}$. Non-small-cell lung cancer carrying an EGFR mutation was much better controlled with gefitinib, an epidermal growth factor receptor (EGFR) tyrosine kinase inhibitor
(TKI), than with chemotherapy, but chemotherapy was superior if the mutation was absent. Targeted therapies are currently used for metastatic NSCLC with mutations in EGFR, $A L K, R O S 1$, and $B R A F^{5}$. For patients with mutation-driven lung cancers, median survival now approaches 3 years, a dramatic improvement compared with the 12 months historically seen ${ }^{5}$. Although no evidence yet supports the use of targeted therapies for patients with SCLC or stages I-III NSCLC, such therapies have revolutionized the management of metastatic NSCLC. However, in North America, targetable driver mutations are currently found in only approximately $25 \%$ of patients. The need for improved outcomes for the other $75 \%$ has driven innovation and the emergence of immunotherapy ("immuno-oncology") in the treatment of lung cancer ${ }^{5}$.

As explored in depth by Esfahani and colleagues ${ }^{6}$ in their article about the mechanisms of immuno-oncology in this supplement, immunotherapy attempts to recruit the immune system to attack cancer by simultaneously overcoming the tumour's ability to evade the immune system and by upregulating the intensity of immune responses ${ }^{7}$. In lung cancer, the rationale for immunotherapy as a therapeutic option rests on lung cancer's mechanisms of immune evasion. Lung cancer evades immune responses by reducing antigen presentation, secreting 
immune-inhibitory cytokines, upregulating immune checkpoints, and stimulating immunosuppressive cell subsets $^{8}$. Antigen presentation is reduced through decreased expression and haplotype loss of human leukocyte antigen. The cytokine transforming growth factor beta is elevated in the serum of lung cancer patients. Transforming growth factor beta inhibits proliferation of normal bronchial epithelial cells, drives conversion of cytotoxic T cells to immunosuppressive $T$ regulatory cells, and possibly enhances cell death for activated $\mathrm{T}$ cells. The latter mechanism also helps to explain the proliferation of $\mathrm{T}$ regulatory cells, which prevent effective immune responses to tumour antigens. Finally, increased presentation of the co-stimulatory molecule PD-Ll on lung cancer cells downregulates and inhibits antitumour immune responses ${ }^{8}$. Recently adopted agents (Figure 1) have targeted those co-stimulatory molecules primarily through blockade of the interaction between PD-1 and PD-L1 or PD-L2?

\section{REVIEW}

\section{Historical Failure of Immuno-oncology in Lung Cancer}

Multiple randomized controlled trials (RCTs) have investigated immunotherapies in lung cancer. Those therapies have included cytokines, vaccines, and immune checkpoint inhibitors (ICIs).

In 1994, a small RCT involving 60 patients with advanced NSCLC compared interleukin 2 plus melatonin with cisplatin-etoposide ${ }^{9}$. Interleukin 2 promotes the differentiation of immature $\mathrm{T}$ cells into effector $\mathrm{T}$ cells, thus attempting to overcome immunosuppression. The response rate to immunotherapy was similar to that with chemotherapy, but survival at 1 year was superior. A larger trial of chemotherapy with or without interleukin 2 showed no difference in response, median progression-free survival (PFS), or 1-year survival ${ }^{10}$.

Four vaccines have aimed to improve antigen presentation and to drive an immune response. Maintenance vaccination with Bec2/bacillus Calmette-Guérin did not improve survival in SCLC patients ${ }^{11}$. Similarly, tecemotide, directed against the mucin 1 glycoprotein, failed to improve overall survival (OS) in patients with unresectable stage III NSCLC when given after chemoradiotherapy ${ }^{12}$. In the setting of resected (stages IB-IIIA) MAGE-A3-positive NSCLC,

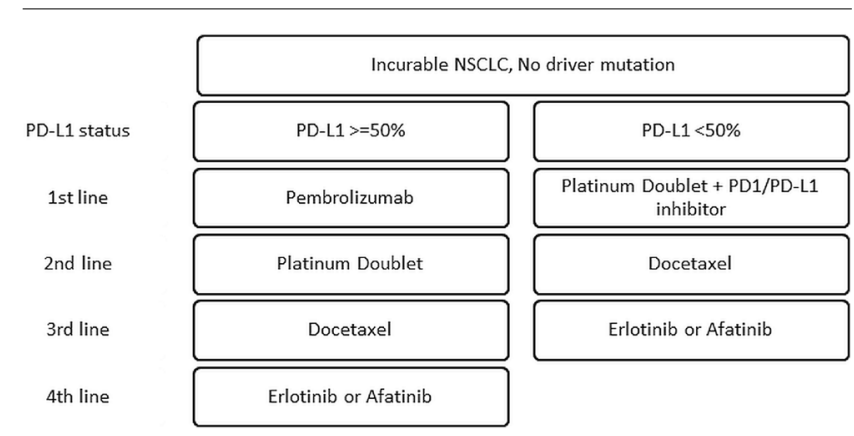

FIGURE 1 Treatment for advanced non-small-cell lung cancer (NSCLC) without driver mutations and with good performance status. adjuvant therapy with a MAGE-A3-directed immunotherapy did not affect disease-free survival ${ }^{13}$. The allogeneic tumour cell vaccine belagenpumatucel-L did not improve median survival ${ }^{14}$. In each of those NSCLC vaccine trials, a hint of benefit was observed, with modest improvements in outcomes, but without the primary survival metrics ever meeting statistical significance.

Finally, in an attempt to overcome immunosuppressive checkpoints, the CTLA-4-directed ICI ipilimumab, when compared with chemotherapy, failed to improve os in patients with advanced NSCLC whether given alone or with chemotherapy ${ }^{15,16}$. Fortunately, recent trials focused on ICIs targeting the PD-1/PD-L1 interaction (Table I) have demonstrated clinically meaningful benefit for patient survival and have transformed the treatment of lung cancer.

\section{Biomarkers}

Building on the success of treatment directed at driver mutations, multiple biomarkers have been investigated to predict benefit with PD-1/PD-L1 ICIs ${ }^{17}$. (Biomarkers related to ICIs are discussed in depth elsewhere in this special issue.) To date, the primary biomarker used for lung cancer has been PD-L1 $1^{7,17}$. Multiple assays to measure PD-L1 in histologic samples of lung cancer are available. Although the cut-offs for positivity vary with the test, each assay identifies negative and positive subgroups that translate into patient populations that are, respectively, less and more likely to respond to ICIs. Most trials in the relapsed or refractory setting identified an os benefit in the full intention-to-treat population. Upon subgroup analysis, though, higher levels of PD-L1 were associated with superior response and greater improvements in median os with PD-1 or PD-L1 inhibitors than with docetaxel. The randomized trials of pembrolizumab uniquely excluded patients who did not express PD-L1. The PD-Ll companion diagnostic result therefore affects the use of pembrolizumab, most importantly in the first-line setting, in which pembrolizumab, compared with platinum doublet chemotherapy, improves os for patients with NSCLC and PD-L1 expression of $50 \%$ or greater ${ }^{18}$.

Absence of PD-L1 expression does not conclusively identify patients who will not benefit from immunotherapy, leading to investigation of many other biomarkers ${ }^{7,17}$. Tumour mutational burden (TMB) is the second most prominently investigated biomarker in patients with lung cancer. High TMB is associated with both a higher response rate and better os for patients treated with nivolumab-ipilimumab ${ }^{19}$. PD-L1 and Tмв appear to be independently predictive. Their levels are not highly correlated, and they potentially provide complementary information for decision-making. Even basic patient characteristics appear to help predict who will benefit from immunotherapy. Female sex might be associated with poorer survival and less benefit ${ }^{20}$. Never-smoker status also appears to be linked to less benefit $^{21,22}$. Those trends are possibly driven by the prevalence of $E G F R$ and $A L K$ mutations in women and never-smokers. Reports from additional trials will clarify the issue.

\section{Previously Treated Advanced NSCLC}

Two large RCTs established checkpoint inhibitor therapy with nivolumab as a soc for the second-line treatment of advanced NSCLC. CheckMate 017 randomized 272 patients 
TABLE I Immune checkpoint inhibitors with reported phase III trial results for lung cancer

\begin{tabular}{lc}
\hline \multicolumn{1}{c}{ Agent } & Target \\
\hline Ipilimumab & CTLA-4 \\
\hline Nivolumab & PD-1 \\
\hline Pembrolizumab & PD-1 \\
Atezolizumab & PD-L1 \\
\hline Durvalumab & PD-L1 \\
Avelumab & PD-L1 \\
\hline
\end{tabular}

CTLA-4 = cytotoxic T lymphocyte-associated protein 4; PD-1 = programmed cell death $1 ; \mathrm{PD}-\mathrm{L} 1=$ programmed cell death ligand 1.

with advanced squamous-cell carcinoma to either nivolumab or docetaxel after progression on platinum doublet chemotherapy ${ }^{23}$. The os was 9.2 months for nivolumab compared with 6.0 months for docetaxel, representing a $41 \%$ decrease in risk of death [hazard ratio (HR): $0.59 ; 95 \%$ confidence interval (CI): 0.44 to 0.79 ]. The response rate and PFS were both superior in the nivolumab arm, and benefit was seen regardless of PD-L1 level. CheckMate 057 randomized 582 patients with advanced nonsquamous NSCLC to either nivolumab or docetaxel ${ }^{24}$. As in CheckMate 017, all patients had progressed on first-line platinum doublet chemotherapy. Patients with EGFR-and $A L K$-mutated NSCLC had to have progressed on appropriate TKI therapy as well. The os was 12.2 months for nivolumab compared with 9.4 months for docetaxel, representing a $27 \%$ decrease in the risk of death (HR: $0.73 ; 96 \%$ CI: 0.59 to 0.89 ). The response rate was not significantly different between the groups, but the PFs favoured nivolumab at 1 year (19\% vs. $8 \%)$. In a subgroup analysis, os was superior with nivolumab only in patients with a PD-L1 expression level of at least $1 \%$. Durable responses were noted in both trials, with $37 \%$ and $34 \%$ of confirmed responders having squamous and nonsquamous tumours respectively experiencing ongoing responses at 2 years ${ }^{25}$. Rates of treatment-related adverse events were lower with nivolumab than with docetaxel ${ }^{23,24}$.

Pembrolizumab has also demonstrated efficacy in the second-line treatment of advanced NSCLC. The KEYNOTE-010 trial randomized 1034 patients to pembrolizumab at two dose levels $\left(2 \mathrm{mg} / \mathrm{kg}\right.$ and $10 \mathrm{mg} / \mathrm{kg}$ ) or to docetaxel ${ }^{26}$. Patients with squamous and nonsquamous tumours were enrolled; however, PD-L1 expression had to be $1 \%$ or greater. All patients had progressed on first-line platinum doublet therapy, and those with driver mutations had also progressed on appropriate TKI therapy. The os was 10.4 months for pembrolizumab $2 \mathrm{mg} / \mathrm{kg}, 12.7$ months for pembrolizumab $10 \mathrm{mg} / \mathrm{kg}$, and 8.5 months for docetaxel. Survival was significantly better for each pembrolizumab group than for the docetaxel group (HR: 0.71 for $2 \mathrm{mg} / \mathrm{kg}$ and 0.61 for $10 \mathrm{mg} / \mathrm{kg}$ ), without a significant difference between the pembrolizumab dose levels. No significant difference in PFS was observed between the treatment arms. Pembrolizumab efficacy was greatest in patients with a PD-L1 tumour proportion score of $50 \%$ or greater.

The OAK trial randomized patients with previously treated advanced NSCLC to either atezolizumab or docetaxel regardless of PD-L1 status ${ }^{27}$. Patients could have received up to 2 previous lines of treatment, with 1 being platinum doublet chemotherapy. Patients with EGFR or ALK mutations had received prior TKI therapy as well as a platinum doublet. The os was 13.8 months for atezolizumab compared with 9.6 months for docetaxel (HR: 0.73; 95\% CI: 0.62 to 0.87 ). Patients with high tumour PD-L1 expression experienced the greatest benefit from atezolizumab; however, os was improved even when PD-L1 expression was lacking. Benefit was observed across histologic subtypes.

Not all trials using PD-1 and PD-L1 checkpoint inhibitors for the second-line treatment of advanced NSCLC have yielded positive results. Avelumab, an anti-PD-L1 monoclonal antibody, was compared with docetaxel in the JAVELIN Lung 200 trial $^{28}$. The os was not significantly different between the avelumab and docetaxel groups, even in the subgroup with positive tumour PD-L1 expression. High post-study use of ICIs and the non-blinded design of the trial might have affected the results. Table II summarizes the second-line ICI trials.

Currently, ICIs are now well established as a soc for the second-line treatment of advanced NSCLC, but there are no data to suggest that one ICI is superior to another in that setting. No head-to-head RCTs have been conducted. Recent network meta-analyses using data from studies of nivolumab, pembrolizumab, and atezolizumab did not demonstrate significant evidence of survival differences between those drugs ${ }^{29,30}$. Factors that could influence ICI selection include access, dosing schedule, cost, and PD-L1 expression.

\section{First-Line Treatment of Advanced NSCLC}

Several phase III RCTs have explored ICIs in the first-line treatment of advanced NSCLC (Table III). The KEYNOTE-024 trial randomized patients with previously untreated advanced NSCLC without EGFR or ALK mutations to either pembrolizumab or platinum doublet chemotherapy ${ }^{18}$. Only patients with a PD-L1 tumour proportion score of $50 \%$ or greater were enrolled. The initial analysis demonstrated a significant improvement in PFs for pembrolizumab compared with chemotherapy (10.3 months vs. 6.0 months). The estimated os rate at 6 months was $80.2 \%$ for pembrolizumab compared with $72.4 \%$ for chemotherapy, with response rates also favouring pembrolizumab. An updated analysis confirmed those results, showing a median os of 30.0 months in the pembrolizumab arm compared with 14.2 months in the chemotherapy arm (HR: 0.63; 95\% CI: 0.47 to 0.86$)^{38}$. Thus, KEYNOTE- 024 defined a new SOC for advanced NSCLC with a PD-L1 tumour proportion score of $50 \%$ or greater.

CheckMate 026 randomized patients with treatmentnaïve advanced NSCLC without EGFR or ALKmutations and with a PD-L1 level of $1 \%$ or greater to either nivolumab or platinum doublet chemotherapy ${ }^{39}$. Despite the benefit seen with nivolumab in second-line treatment, os was not superior to that with chemotherapy in the first-line setting. Treatment with nivolumab resulted in an os of 14.4 months compared with 13.2 months with chemotherapy. There was also no significant improvement in PFS or response rate. Interestingly, an exploratory analysis of TMB demonstrated an increased response rate and improved PFs for 
TABLE II Randomized trials of immune checkpoint inhibitors in previously treated advanced non-small-cell lung cancer

\begin{tabular}{|c|c|c|c|c|c|c|c|}
\hline \multirow{2}{*}{$\begin{array}{c}\text { Reference } \\
\text { (trial name) }\end{array}$} & \multirow{2}{*}{$\begin{array}{l}\text { Enrolment } \\
\text { period }\end{array}$} & \multirow[t]{2}{*}{ Treatment arms } & \multirow{2}{*}{$\begin{array}{l}\text { Pts } \\
\text { (n) }\end{array}$} & \multicolumn{3}{|c|}{ Median OS } & \multirow{2}{*}{$\begin{array}{c}\text { Grade } 3 \text { or greater } \\
\text { treatment-related } \\
\text { adverse events }(\%)\end{array}$} \\
\hline & & & & (months) & HR & $\mathrm{Cl}$ & \\
\hline \multirow{2}{*}{$\begin{array}{l}\text { Brahmer et al., } 2015^{23} \\
\text { (CheckMate 057, } \\
\text { nonsquamous) }\end{array}$} & \multirow[t]{2}{*}{ 2012-2013 } & Nivolumab & 287 & 12.2 & \multirow[t]{2}{*}{0.73} & \multirow{2}{*}{$\begin{array}{c}96 \%: \\
0.59 \text { to } 0.89\end{array}$} & 10 \\
\hline & & Docetaxel & 268 & 9.4 & & & 54 \\
\hline \multirow{2}{*}{$\begin{array}{l}\text { Herbst et al., } 2016^{26} \\
\text { (OAK, all) }\end{array}$} & \multirow[t]{2}{*}{ 2014-2015 } & Atezolizumab & 425 & 13.8 & \multirow[t]{2}{*}{0.73} & \multirow{2}{*}{$\begin{array}{c}95 \%: \\
0.62 \text { to } 0.87\end{array}$} & 15 \\
\hline & & Docetaxel & 425 & 9.6 & & & 43 \\
\hline \multirow{3}{*}{$\begin{array}{l}\text { Horn et al., } 2017^{25} \\
\text { (KEYNOTE-010, } \\
\text { PD-L1 } \geq 1 \%)\end{array}$} & \multirow[t]{3}{*}{ 2013-2015 } & $\begin{array}{l}\text { Pembrolizumab } \\
2 \mathrm{mg} / \mathrm{kg}\end{array}$ & 345 & 10.4 & & & 13 \\
\hline & & $\begin{array}{l}\text { Pembrolizumab } \\
10 \mathrm{mg} / \mathrm{kg}\end{array}$ & 346 & 12.7 & & & 16 \\
\hline & & Docetaxel & 343 & 8.5 & & & 35 \\
\hline \multirow{2}{*}{$\begin{array}{l}\text { Rittmeyer et al., } 2017^{27} \\
\text { (JAVELIN Lung 200, } \\
\text { PD-L1 } \geq 1 \% \text { ) }\end{array}$} & \multirow[t]{2}{*}{$2015-2017$} & Avelumab & 264 & 11.4 & \multirow[t]{2}{*}{0.90} & \multirow{2}{*}{$\begin{array}{c}96 \%: \\
0.72 \text { to } 1.12\end{array}$} & 10 \\
\hline & & Docetaxel & 265 & 10.3 & & & 49 \\
\hline \multirow{2}{*}{$\begin{array}{l}\text { Huang et al., } 2018^{22} \\
\text { (CheckMate 017, squamous) }\end{array}$} & \multirow[t]{2}{*}{ 2012-2013 } & Nivolumab & 135 & 9.2 & \multirow[t]{2}{*}{0.59} & \multirow{2}{*}{$\begin{array}{c}95 \%: \\
0.44 \text { to } 0.79\end{array}$} & 7 \\
\hline & & Docetaxel & 137 & 6.0 & & & 57 \\
\hline
\end{tabular}

$\mathrm{HR}=$ hazard ratio; $\mathrm{Cl}=$ confidence interval.

nivolumab in patients with a higher TMB. Patients with the highest TMB and PD-L1 expression experienced the highest response rate. Several hypotheses have been put forward for why first-line nivolumab failed while pembrolizumab succeeded, including differences in the PD-Ll cut-off for eligibility, the PD-L1 test used, and the criteria established for corticosteroid use and prior radiotherapy ${ }^{18,39}$. Differences between the treatment arms in CheckMate 026 might also have contributed, including fewer patients with high $\mathrm{PD}-\mathrm{L} 1$ in the nivolumab arm and better baseline prognosis characteristics in the chemotherapy $\operatorname{arm}^{31,39}$.

With the success of first-line pembrolizumab in patients with a PD-L1 status of $50 \%$ or greater, the KEYNOTE-042 trial explored whether first-line pembrolizumab was superior to platinum doublet chemotherapy in patients with advanced NSCLC and a PD-L1 tumour proportion score of $1 \%$ or greater ${ }^{34}$. In patients meeting those criteria, os was 16.7 months for pembrolizumab compared with 12.1 months for chemotherapy (HR: 0.81; 95\% CI: 0.71 to 0.93 ). The benefit with pembrolizumab was greater with higher PD-L1 proportion scores. Although those results are promising, an exploratory analysis in patients with a PD-L1 proportion score of $1 \%-49 \%$ did not show a significant benefit with pembrolizumab compared with chemotherapy, suggesting that the survival benefit seen in the overall study population was driven primarily by patients with high PD-Ll expression.

The combination of chemotherapy and immunotherapy has been explored in multiple RCTs. The KEYNOTE- 407 trial randomized 559 patients with treatment-naïve advanced squamous-cell carcinoma of the lung to carboplatin-taxane (paclitaxel or nab-paclitaxel) plus either pembrolizumab or placebo, regardless of PD-L1 expression ${ }^{36}$. Patients received 4 cycles of chemotherapy and up to 35 cycles of pembrolizumab or placebo. The os was 15.9 months in the pembrolizumab-chemotherapy arm compared with 11.3 months in the chemotherapy-placebo arm (HR: $0.64 ; 95 \%$ CI: 0.49 to 0.85 ). A survival benefit was seen at all levels of PD-L1 expression. The similarly designed KEYNOTE-189 trial randomized 616 patients with treatment-naïve advanced nonsquamous NSCLC and no $A L K$ or $E G F R$ mutation to 4 cycles of platinum-pemetrexed followed by pemetrexed maintenance plus pembrolizumab or placebo ${ }^{32}$. Median os was not reached in the chemotherapy-pembrolizumab arm; it was 11.3 months in the chemotherapy-placebo arm (HR: 0.49 ; $95 \%$ CI: 0.38 to $0.64)$. As in the KEYNOTE- 407 trial, a benefit was seen regardless of PD-L1 expression level. Those results established the combination of chemotherapy and pembrolizumab as a sOC in the first-line treatment of advanced NSCLC; however, whether chemotherapy is necessary in patients with PD-L1 expression of $50 \%$ or greater remains unclear.

The IMpower150 trial explored the combination of chemotherapy, bevacizumab, and atezolizumab for patients with treatment-naïve advanced nonsquamous NSCLC ${ }^{37}$. That 3-arm trial randomized patients to carboplatinpaclitaxel chemotherapy plus bevacizumab, chemotherapy plus bevacizumab and atezolizumab, or chemotherapy plus atezolizumab. Currently, the published comparison is for chemotherapy-bevacizumab compared with chemotherapy-bevacizumab-atezolizumab. For patients without an EGFR or $A L K$ mutation, os was 19.2 months in the chemotherapy-bevacizumab-atezolizumab arm and 14.7 months in the chemotherapy-bevacizumab arm (HR: 0.78 ; 95\% CI: 0.64 to 0.96 ). Although carboplatinpaclitaxel-bevacizumab is not a commonly used regimen for nonsquamous NSCLC in Canada, results validate the efficacy of chemotherapy and ICI combinations. Results from trials that are comparing atezolizumab combined with various platinum doublet backbones (IMpower130, 131, and 132) were presented in 2018, each suggesting a benefit for chemotherapy plus immunotherapy compared 
TABLE III Randomized trials of immune checkpoint inhibitors in treatment-naïve advanced non-small-cell lung cancer

\begin{tabular}{|c|c|c|c|c|c|c|}
\hline $\begin{array}{c}\text { Reference } \\
\text { (trial name) }\end{array}$ & $\begin{array}{c}\text { Median } \\
\text { follow-up } \\
\text { (months) }\end{array}$ & Treatment arms & HR & $95 \% \mathrm{CI}$ & $\begin{array}{c}\text { Median } \\
\text { OS } \\
\text { (months) }\end{array}$ & $\begin{array}{c}\text { Grade } 3 \text { or greater } \\
\text { treatment-related } \\
\text { adverse events }(\%)\end{array}$ \\
\hline \multirow{2}{*}{$\begin{array}{l}\text { Reck et al., 2016 } \\
\quad \text { (CheckMate 227, high TMB) }\end{array}$} & \multirow{2}{*}{$\begin{array}{l}\text { Minimum } \\
\quad 11.2\end{array}$} & Nivolumab-ipilimumab & \multirow[t]{2}{*}{ NR } & & \multirow[t]{2}{*}{$N R$} & 31.2 \\
\hline & & Platinum doublet & & & & 36.1 \\
\hline \multirow{2}{*}{$\begin{array}{l}\text { Remon et al., 201731 } \\
\text { (KEYNOTE-042, PD-L1 1 1\%) }\end{array}$} & \multirow[t]{2}{*}{12.8} & Pembrolizumab & \multirow[t]{2}{*}{0.81} & \multirow[t]{2}{*}{0.71 to 0.93} & 16.7 & 17.8 \\
\hline & & Platinum doublet & & & 12.1 & 41.0 \\
\hline \multirow[t]{3}{*}{$\begin{array}{l}\text { Gandhi et al., } 2018^{32} \\
\text { (IMpower150, nonsquamous) }\end{array}$} & \multirow[t]{3}{*}{20.0} & $\begin{array}{l}\text { (A) Paclitaxel-carboplatin- } \\
\text { bevacizumab-atezolizumab }\end{array}$ & \multirow{3}{*}{$\begin{array}{l}\text { A vs. C: } \\
0.78 \\
\text { B vs. C: } \\
0.88\end{array}$} & \multirow[t]{3}{*}{0.64 to 0.96} & 19.2 & \multirow[t]{2}{*}{58.5} \\
\hline & & $\begin{array}{l}\text { (B) Paclitaxel-carboplatin- } \\
\text { atezolizumab }\end{array}$ & & & 19.4 & \\
\hline & & $\begin{array}{c}\text { (C) Paclitaxel-carboplatin- } \\
\text { bevacizumab }\end{array}$ & & & 14.7 & 50.0 \\
\hline \multirow[t]{2}{*}{$\begin{array}{l}\text { Jotte et al., 2018 } \\
\text { (IMpower130) }\end{array}$} & \multirow[t]{2}{*}{19.0} & $\begin{array}{l}\text { Nab-paclitaxel-carboplatin- } \\
\text { atezolizumab }\end{array}$ & \multirow[t]{2}{*}{0.79} & \multirow[t]{2}{*}{0.64 to 0.98} & 18.6 & 74.9 \\
\hline & & Nab-paclitaxel-carboplatin & & & 13.9 & 60.7 \\
\hline \multirow{2}{*}{$\begin{array}{l}\text { Lopes et al., } 2018^{34} \\
\text { (KEYNOTE-407, squamous) }\end{array}$} & \multirow[t]{2}{*}{7.8} & Chemotherapy-pembrolizumab & \multirow[t]{2}{*}{0.64} & \multirow[t]{2}{*}{0.49 to 0.85} & 15.9 & 69.8 \\
\hline & & Chemotherapy-placebo & & & 11.3 & 68.2 \\
\hline \multirow[t]{3}{*}{$\begin{array}{l}\text { Papadimitrakopoulou et al., } 2018^{35} \\
\text { (IMpower131) }\end{array}$} & \multirow[t]{3}{*}{17.1} & $\begin{array}{l}\text { (A) Nab-paclitaxel-carboplatin- } \\
\text { atezolizumab }\end{array}$ & \multirow[t]{3}{*}{$\begin{array}{l}\text { B vs. C: } \\
0.96\end{array}$} & \multirow[t]{3}{*}{0.78 to 1.18} & 14.0 & \\
\hline & & $\begin{array}{l}\text { (B) Paclitaxel-carboplatin- } \\
\text { atezolizumab }\end{array}$ & & & NR & 69 \\
\hline & & (C) Nab-paclitaxel-carboplatin & & & 13.9 & 58 \\
\hline \multirow{2}{*}{$\begin{array}{l}\text { Paz-Ares et al., } 2018^{36} \\
\text { (KEYNOTE-189, nonsquamous) }\end{array}$} & \multirow[t]{2}{*}{10.5} & Chemotherapy-pembrolizumab & \multirow[t]{2}{*}{0.49} & \multirow[t]{2}{*}{0.38 to 0.64} & NR & 67.2 \\
\hline & & Chemotherapy-placebo & & & 11.3 & 65.8 \\
\hline \multirow[t]{2}{*}{$\begin{array}{l}\text { Socinski et al., } 2018^{37} \\
\text { (IMpower132) }\end{array}$} & \multirow[t]{2}{*}{14.8} & $\begin{array}{l}\text { Pemetrexed-platinum CTx- } \\
\text { atezolizumab }\end{array}$ & \multirow[t]{2}{*}{0.81} & \multirow[t]{2}{*}{0.64 to 1.03} & 18.1 & 58 \\
\hline & & Pemetrexed-platinum CTx & & & 13.6 & 42 \\
\hline \multirow{2}{*}{$\begin{array}{l}\text { Tan et al., } 2018^{30} \text {, and } \\
\text { Camidge et al., } 2019^{17} \\
\text { (KEYNOTE-024, PD-L1 } \geq 50 \%\end{array}$} & 25.2 & Pembrolizumab & 0.63 & 0.47 to 0.86 & 30.0 & 26.6 \\
\hline & & Platinum doublet & & & 14.2 & 53.3 \\
\hline Reck et al., $2019^{38}$ & 14.0 & Nivolumab & 1.02 & 0.80 to 1.30 & 14.4 & 18 \\
\hline (CheckMate 026, PD-LI & & Platinum doublet & & & 13.2 & 51 \\
\hline
\end{tabular}

$\mathrm{HR}=$ hazard ratio; $\mathrm{Cl}=$ confidence interval; $\mathrm{OS}=$ overall survival; $\mathrm{TMB}=$ tumour mutational burden; $\mathrm{NR}=$ not reported.

with chemotherapy alone $e^{33,35,40}$. The full publications from those trials had not been released at time of writing, and so details are not included for those publications.

Combination immunotherapy is also being studied in advanced NSCLC. CheckMate 227 is a complex multipart study that randomized patients with PD-Ll expression of $1 \%$ or greater to nivolumab-ipilimumab, nivolumab monotherapy, or platinum doublet chemotherapy ${ }^{19}$. Patients with PD-Ll expression less than $1 \%$ were randomized to nivolumab-ipilimumab, nivolumab plus chemotherapy, or chemotherapy alone. The protocol was amended to add a co-primary endpoint of PFS for nivolumab-ipilimumab compared with chemotherapy in patients with a high TMB (defined as at least 10 mutations per megabase). The results of the biomarker analysis showed that the PFS in the high TMB subgroup was 7.2 months with nivolumab-ipilimumab and 5.5 months with chemotherapy (HR: $0.58 ; 97.5 \%$ CI: 0.41 to 0.81 ). A benefit in PFS was seen regardless of PD-L1 expression. The os results were immature, but encouraging. Although those results are promising, it remains to be seen whether combination immunotherapy is superior to monotherapy or the combination of immunotherapy and chemotherapy. Further trials are ongoing.

\section{Duration of Treatment}

The optimal duration of treatment with ICIs for patients with advanced NSCLC remains an open question. Trials of nivolumab and atezolizumab in previously treated patients were designed with unlimited continuation of treatment until disease progression or lack of tolerance ${ }^{23,24,27}$. On the other hand, trials of pembrolizumab stopped treatment after a maximum of 2 years ${ }^{26}$. After 43 months' 
median follow-up in KEYNOTE-010, 79 of 690 patients received pembrolizumab for the maximal 2 years, and 25 (32\%) developed progressive disease after stopping ${ }^{41}$. Of the 14 patients re-treated with pembrolizumab, 11 experienced a partial re-response or disease stabilization. The most rigorous study to assess treatment duration (CheckMate 153) randomized patients still receiving nivolumab after 1 year to observation or continuation ${ }^{42}$. Of 1245 initial patients, 220 were still receiving treatment at 1 year, and 163 with stable or responding NSCLC were included in an efficacy analysis. The PFS was superior in the continuation arm (not reached vs. 10.3 months), and a statistically nonsignificant trend toward better os was observed (HR: 0.63; 95\% CI: 0.33 to 1.20$)$. Of the 87 patients who stopped nivolumab, 43 (49\%) developed progressive disease. In 34 patients who received nivolumab re-treatment, the median duration of re-treatment at data cut-off was 3.8 months (range: 0.1-17.5 months). The data available to judge duration of immunotherapy treatment and the value of re-treatment are inadequate to reach firm conclusions. It is possible that 1 year is too early a stopping point. CheckMate 153 does not demonstrate a statistically significant survival benefit, although it was underpowered for that outcome. Currently, the best recommendation is to follow the trial protocols for each drug.

\section{Special Populations}

Patients whose NSCLC carries an EGFR or ALK mutation appear to benefit much less from ICIs than do patients with a wild-type profile. Subgroup analyses from landmark RCTs establishing monotherapy with nivolumab, pembrolizumab, or atezolizumab showed no evidence of improved survival in patients with EGFR mutations ${ }^{23,24,26,27}$. A meta-analysis of EGFR-mutated NSCLC from four trials showed no benefit (HR: 1.11 ; 95\% CI: 0.80 to 1.53), reducing the likelihood that the lack of benefit is simply attributable to a small sample size ${ }^{22}$. Patients with $A L K$-translocated NSCLC were also included in those trials, but no publications have reported on that subgroup. Patients with EGFR and $A L K$ mutations were excluded from most first-line immunotherapy trials, but the IMpower150 trial, discussed earlier, included 80 patients with EGFR-mutated NSCLC and 34 with $A L K$-translocated NSCLC ${ }^{37}$. When combined, those subgroups experienced superior PFs with the inclusion of atezolizumab (HR: $0.59 ; 95 \% \mathrm{CI}: 0.37$ to 0.94 ). That benefit raises the question of adding immunotherapy to targeted therapy-the typical first-line standard for targetable mutations. However, combinations of ICIs with gefitinib and crizotinib have caused severe hepatotoxicity, and combinations with osimertinib have caused severe pneumonitis, curtailing enthusiasm for those combinations ${ }^{43,44}$. The relative insensitivity of $E G F R$ - and $A L K$-mutated NSCLC to immunotherapy might be related to a comparatively low TMB, lower levels of PD-L1, and the ongoing presence of a growth signal once targeted therapy has been stopped. Interestingly, the exception to that trend of decreased efficacy with driver mutations is seen with $K R A S$-mutated NSCLC. In individual RCTs and systematic reviews alike, the likelihood of response and improved os are higher when $K R A S$-mutated NSCLC is treated with ICIs rather than with chemotherapy ${ }^{21,22}$. The reason that KRAS-mutated NSCLC behaves differently from EGFR-mutated NSCLC upon exposure to immunotherapy remains an area of active research.

Concerns have also been raised about benefit from ICIs for lung cancer in older adults and in patients with a poor performance status (PS). Older adults experience changes in the immune system that might blunt immune responsiveness ${ }^{45}$. A meta-analysis of six trials showed a net survival advantage for immunotherapy compared with chemotherapy in patients 65 or more years of age (HR: $0.72 ; 95 \%$ CI: 0.58 to 0.89$)^{22}$. In a real-world setting, older patients $(65-75$ years and $>75$ years) experience outcomes similar to those in patients on trials, the median os being approximately 12 months ${ }^{46,47}$. Patients with a poor Ps were not included in the landmark clinical trials. A phase II trial of nivolumab for such patients (Eastern Cooperative Oncology Group PS 2-4) with advanced NSCLC showed a PS improvement in $29 \%$ of patients and an os of 9.3 months in those with EGFR wild-type NSCLC ${ }^{48}$. Those data suggest that patients with a poor PS can still benefit; however, the trial included only 33 patients, and so caution and further research are still required before this approach can be adopted.

\section{Stage III NSCLC}

The soc treatment in stage III NSCLC is concurrent chemoradiotherapy. The chemotherapy component typically involves one of three regimens: cisplatin-etoposide, cisplatin-vinorelbine, or carboplatin-paclitaxel ${ }^{5}$. Consolidative chemotherapy does not appear to improve survival after concurrent chemoradiotherapy ${ }^{49}$. Although treatment for metastatic NSCLC has evolved markedly since 2009, no major advances in systemic therapy for stage III NSCLC had been made until the PACIFIC trial. That trial randomized 713 patients to receive either standard chemoradiotherapy plus placebo or soc plus 1 year of consolidative durvalumab. Interim analysis showed that, compared with Soc alone, the addition of durvalumab was associated with superior 24 -month survival ( $66.3 \%$ vs. $55.6 \%)$ and os (HR: 0.68 ; 99.73\% CI: 0.47 to 0.997$)^{50}$. Benefit appeared to be similar in most subgroups, including different levels of PD-L1, with the main uncertainty being efficacy in patients with EGFR-mutated NSCLC. From a safety perspective, grades 3-4 toxicities were observed in $29.9 \%$ of patients in the durvalumab group compared with $26.1 \%$ of those in the placebo group. Most importantly, the rate of pneumonitis was $33.9 \%$ for the durvalumab group compared with $24.8 \%$ for the placebo group, but grades 3-4 pneumonitis occurred in only $3.4 \%$ and $2.6 \%$ respectively ${ }^{51}$. The PACIFIC trial thus created a new soc for patients with stage III NSCLC.

\section{SCLC}

In patients with SCLC, results for immunotherapy, including PD1/PD-L1 inhibitors, have been more mixed than they have for patients with NSCLC. The single-arm KEYNOTE-158 trial assessed the effect of pembrolizumab monotherapy in patients with extensive-stage SCLC after failure of standard therapy ${ }^{52}$. Encouragingly, patients showing PD-L1 positivity responded to pembrolizumab $35.7 \%$ of the time and had a median os of 14.9 months. The benefit in PD-L1-negative SCLC was minimal. Additional trials for treatment-refractory SCLC are ongoing. Combination immunotherapy with nivolumab-ipilimumab, tested in the 
CheckMate 032 trial, was associated with a response rate of $23 \%$ and an os duration of 7.7 months $^{53}$. The phase III CheckMate 451 trial will provide further clarity about combination immunotherapy in SCLC.

The combination of ICIs with chemotherapy has been more successful to date. The IMpower133 randomized trial demonstrated that adding atezolizumab to carboplatinetoposide for patients with treatment-naïve extensive-stage sCLC improves survival. The os was 12.3 months with atezolizumab compared with 10.3 months with chemotherapy alone (HR: $0.70 ; 95 \%$ CI: 0.54 to 0.91$)^{54}$. The rate of treatment-related adverse events was minimally different. IMpower133 is changing the 30 -year soc for systemic therapy in extensive-stage SCLC.

\section{FUTURE OF IMMUNO-ONCOLOGY IN LUNG CANCER}

The development of immuno-oncology, especially ICIs, has revolutionized the treatment of lung cancer and will continue to drive therapeutic evolution. The next logical step in the use of ICIs for patients with lung cancer is to move their use to patients with earlier-stage disease. Currently, ongoing trials are investigating whether there is a benefit to providing ICI treatment in either the adjuvant or neoadjuvant setting. Trials examining additional combinations of approved drugs, such as platinum doublet chemotherapy plus both a PD- 1 inhibitor and a CTLA- 4 inhibitor are also underway. New drugs being assessed include those targeted to inhibitory molecules such as LAG-3 or IDO, co-stimulatory antibodies such OX40, and dual-targeted agents. At the time of writing, a search of ClinicalTrials. gov revealed more than 270 trials currently focused on immunotherapy, immuno-oncology, or checkpoint inhibitors for lung cancer. Immuno-oncology has become one of the pillars of lung cancer treatment and will likely be the paradigm of therapeutic innovation for years to come.

\section{ACKNOWLEDGMENTS}

DED acknowledges the Manitoba Medical Services Foundation, which has provided him with a salary award to protect some of his time for research. No funds were provided from any source for this paper.

\section{CONFLICT OF INTEREST DISCLOSURES}

We have read and understood Current Oncology's policy on disclosing conflicts of interest, and we declare the following interests: DED has participated on advisory boards for AstraZeneca and Merck and has received an honorarium from Boehringer Ingelheim for providing educational content. RAJ has received honoraria from Bristol-Myers Squib, AstraZeneca, Roche Canada, Merck Sharp and Dohme, Novartis Canada Pharmaceuticals Inc., Amgen, and Boehringer Ingelheim, and has consulted or advised for AstraZeneca, Bristol-Myers Squib, Novartis, Pfizer, Roche Canada, Takeda, AbbVie, and Fusion Pharmaceuticals. CHH has no conflicts to disclose.

\section{AUTHOR AFFILIATIONS}

*Department of Medical Oncology and Hematology, CancerCare Manitoba, and Department of Internal Medicine, University of Manitoba, Winnipeg, MB; ${ }^{\dagger}$ Department of Oncology, Juravinski Cancer Centre, Hamilton, ON.

\section{REFERENCES}

1. Canadian Cancer Society's Advisory Committee on Cancer Statistics. Canadian Cancer Statistics 2017. Toronto, ON: Canadian Cancer Society; 2017.

2. Bray F, Ferlay J, Soerjomataram I, Siegel RL, Torre LA, Jemal A. Global cancer statistics 2018: GLOBOCAN estimates of incidence and mortality worldwide for 36 cancers in 185 countries. $C A$ Cancer J Clin 2018;68:394-424.

3. DeVita VT Jr, Lawrence TS, Rosenberg SA, eds. Devita, Hellman, and Rosenberg's Cancer: Principles and Practice of Oncology, 10th edition. Philadelphia, PA: Wolters Kluwer Health; 2015.

4. MokTS, Wu YL, ThongprasertS, etal. Gefitinib or carboplatinpaclitaxel in pulmonary adenocarcinoma. NEnglJMed 2009; 361:947-57.

5. Ettinger DS, Aisner DL, Wood DE, et al. NCCN guidelines insights: non-small cell lung cancer, version 5.2018. J Natl Comp Cancer Netw 2018;16:807-21.

6. Esfahani K, Roudaia L, Buhlaiga N, Del Rincon SV, Papneja N, Miller WH Jr. A review of cancer immunotherapy: from the past, to the present, to the future. Curr Oncol 2020;27:S87-S97.

7. Meyers DE, Bryan PM, Banerji S, Morris DG. Targeting the PD-1/PD-L1 axis for the treatment of non-small-cell lung cancer. Curr Oncol 2018;25:e324-34.

8. Thomas A, Giaccone G. Why has active immunotherapy not worked in lung cancer? Ann Oncol 2015;26:2213-20.

9. Lissoni P, Meregalli S, Fossati V, et al. A randomized study of immunotherapy with low-dose subcutaneous interleukin-2 plus melatonin vs chemotherapy with cisplatin and etoposide as first-line therapy for advanced non-small cell lung cancer. Tumori 1994;80:464-7.

10. Ridolfi L, Bertetto O, Santo A, et al. Chemotherapy with or without low-dose interleukin-2 in advanced non-small cell lung cancer: results from a phase III randomized multicentric trial. Int J Oncol 2011;39:1011-17.

11. Giaccone G, Debruyne C, Felip E, et al. Phase III study of adjuvant vaccination with Bec2/bacille Calmette-Guérin in responding patients with limited-disease small-cell lung cancer (European Organisation for Research and Treatment of Cancer 08971-08971B; Silva Study). J Clin Oncol 2005;23:6854-64.

12. Butts C, Socinski MA, Mitchell PL, etal. Tecemotide (L-BLP25) versus placebo after chemoradiotherapy for stage III nonsmall-cell lung cancer (START): a randomised, double-blind, phase 3 trial. Lancet Oncol 2014;15:59-68.

13. Vansteenkiste JF, Cho BC, Vanakesa T, et al. Efficacy of the MAGE-A3 cancer immunotherapeutic as adjuvant therapy in patients with resected MAGE-A3-positive non-small-cell lung cancer (MAGRIT): a randomised, double-blind, placebocontrolled, phase 3 trial. Lancet Oncol 2016;17:822-35.

14. Giaccone G, Bazhenova LA, Nemunaitis J, et al. A phase III study of belagenpumatucel-L, an allogeneic tumour cell vaccine, as maintenance therapy for non-small cell lung cancer. Eur J Cancer 2015;51:2321-9.

15. Lynch TJ, Bondarenko I, Luft A, et al. Ipilimumab in combination with paclitaxel and carboplatin as first-line treatment in stage ІІІв/IV non-small-cell lung cancer: results from a randomized, double-blind, multicenter phase II study. JClin Oncol 2012;30:2046-54.

16. Govindan R, Szczesna A,Ahn MJ, etal. Phase III trial of ipilimumab combined with paclitaxel and carboplatin in advanced squamous non-small-cell lung cancer.JClin Oncol 2017;35:3449-57.

17. Camidge DR, Doebele RC, Kerr KM. Comparing and contrasting predictive biomarkers for immunotherapy and targeted therapy of NSCLC. Nat Rev Clin Oncol 2019;16:341-55.

18. Reck M, Rodriguez-Abreu D, Robinson AG, et al. on behalf of the KEYNOTE-024 investigators. Pembrolizumab versus chemotherapy for PD-L1-positive non-small-cell lung cancer. N Engl J Med 2016;375:1823-33. 
19. Hellmann MD, Ciuleanu TE, Pluzanski A, et al. Nivolumab plus ipilimumab in lung cancer with a high tumor mutational burden. NEngl J Med 2018;378:2093-104.

20. Wallis CJD, Butaney M, Satkunasivam R, et al. Association of patient sex with efficacy of immune checkpoint inhibitors and overall survival in advanced cancers: a systematic review and meta-analysis. JAMA Oncol 2019;5:529-36.

21. Lee CK, Man J, Lord S, et al. Clinical and molecular characteristics associated with survival among patients treated with checkpoint inhibitors for advanced non-small cell lung carcinoma: a systematic review and meta-analysis. JAMA Oncol 2018;4:210-16.

22. Huang Q, Zhang H, Hai J, et al. Impact of PD-L1 expression, driver mutations and clinical characteristics on survival after anti-PD-1/PD-L1 immunotherapy versus chemotherapy in non-small-cell lung cancer: a meta-analysis of randomized trials. Oncoimmunology 2018;7:e1396403.

23. Brahmer J, Reckamp KL, Baas $\mathrm{P}$, et al. Nivolumab versus docetaxel in advanced squamous-cell non-small-cell lung cancer. N Engl J Med 2015;373:123-35.

24. Borghaei H, Paz-Ares L, Horn L, et al. Nivolumab versus docetaxel in advanced nonsquamous non-small-cell lung cancer. N Engl J Med 2015;373:1627-39.

25. Horn L, Spigel DR, Vokes EE, et al. Nivolumab versus docetaxel in previously treated patients with advanced non-small-cell lung cancer: two-year outcomes from two randomized, openlabel, phase III trials (CheckMate 017 and CheckMate 057). J Clin Oncol 2017;35:3924-33.

26. Herbst RS, Baas P, Kim DW, et al. Pembrolizumab versus docetaxel for previously treated, PD-L1-positive, advanced non-small-cell lung cancer (KEYNOTE-010): a randomised controlled trial. Lancet 2016;387:1540-50.

27. Rittmeyer A, Barlesi F, Waterkamp D, et al. Atezolizumab versus docetaxel in patients with previously treated nonsmall-cell lung cancer (OAK): a phase 3, open-label, multicentre randomised controlled trial. Lancet 2017;389:255-65.

28. Barlesi F, Vansteenkiste J, Spigel D, et al. Avelumab versus docetaxel in patients with platinum-treated advanced nonsmall-cell lung cancer (JAVELIN Lung 200): an open-label, randomised, phase 3 study. Lancet Oncol 2018;19:1468-79.

29. Armoiry X, Tsertsvadze A, Connock M, et al. Comparative efficacy and safety of licensed treatments for previously treated non-small cell lung cancer: a systematic review and network meta-analysis. PLoS One 2018;13:e0199575.

30. Tan PS, Aguiar P Jr, Haaland B, Lopes G. Comparative effectiveness of immune-checkpoint inhibitors for previously treated advanced non-small cell lung cancer-a systematic review and network meta-analysis of 3024 participants. Lung Cancer 2018;115:84-8.

31. Remon J, Besse B, Soria JC. Successes and failures: what did we learn from recent first-line treatment immunotherapy trials in non-small cell lung cancer? BMC Med 2017;15:55. [Erratum in: BMC Med 2017;15:82]

32. Gandhi L, Rodriguez-Abreu D, Gadgeel S, et al. on behalf of the KEYNOTE-189 investigators. Pembrolizumab plus chemotherapy in metastatic non-small-cell lung cancer. $N$ Engl $J$ Med 2018;378:2078-92.

33. Jotte RM, Cappuzzo F, Vynnychenko I, et al. IMpower131: primary PFS and safety analysis of a randomized phase III study of atezolizumab + carboplatin + paclitaxel or nab-paclitaxel vs carboplatin + nab-paclitaxel as $1 \mathrm{~L}$ therapy in advanced squamous NSCLC [abstract LBA9000]. J Clin Oncol 2018;36: [Available online at: https://ascopubs.org/doi/10.1200/ JCO.2018.36.18_suppl.LBA9000; cited 22 February 2020]

34. Lopes G, Wu YL, Kudaba I, et al. Pembrolizumab (pembro) versus platinum-based chemotherapy (chemo) as first-line therapy for advanced/metastatic NSCLC with a PD-L1 tumor proportion score (TPS) $\geq 1 \%$ : open-label, phase 3 KEYNOTE-042 study [abstract LBA4]. JClin Oncol 2018;36:. [Available online at: https://ascopubs.org/doi/10.1200/JCO.2018.36.18_suppl. LBA4; cited 5 September 2019]

35. Papadimitrakopoulou VA, Cobo M, Bordoni R, et al. IMpower132: PFsandsafetyresultswith 1Latezolizumab+carboplatin/ cisplatin + pemetrexed in stage IV non-squamous NSCLC [abstract OA05.07]. J Thorac Oncol 2018;13:.

36. Paz-Ares L, Luft A, Vicente D, et al. on behalf of the KeYNOTE-407 investigators. Pembrolizumab plus chemotherapy for squamous non-small-cell lung cancer. N Engl J Med 2018;379:2040-51.

37. Socinski MA, Jotte RM, Cappuzzo F, et al. on behalf of the IMpower150 study group. Atezolizumab for first-line treatment of metastatic nonsquamous NSCLC. $N$ Engl J Med 2018;378:2288-301.

38. Reck M, Rodriguez-Abreu D, Robinson AG, et al. Updated analysis of KEYNOTE-024: pembrolizumab versus platinumbased chemotherapy for advanced non-small-cell lung cancer with PD-L1 tumor proportion score of $50 \%$ or greater. J Clin Oncol 2019;37:537-46.

39. Carbone DP, Reck M, Paz-Ares L, et al. First-line nivolumab in stage IV or recurrent non-small-cell lung cancer. $N$ Engl $\mathrm{J}$ Med 2017;376:2415-26.

40. Cappuzzo F, McCleod M, Hussein M, et al. IMpower130: progression-free survival (PFS) and safety analysis from a randomised phase III study of carboplatin + nab-paclitaxel $(\mathrm{CnP})$ with or without atezolizumab (atezo) as first-line (1L) therapy in advanced non-squamous NSCLC [abstract LBA53] . Ann Oncol 2018;29:.

41. Herbst RS, Garon EB, Kim D, et al. Long-term follow-up in the KEYNOTE-010 study of pembrolizumab (pembro) for advanced NSCLC, including in patients (pts) who completed 2 years of pembro and pts who received a second course of pembro [abstract LBA4]. Ann Oncol 2018;29:.

42. Spiegel DR, McLeod M, Hussain MA, et al. Randomized results of fixed-duration (1-yr) vs continuous nivolumab in patients (pts) with advanced non-small cell lung cancer (NSCLC) [abstract 1297O]. Ann Oncol 2017;28:.

43. Chih-Hsin Yang J, Shepherd FA, Kim DW, et al. Osimertinib plus durvalumab versus osimertinib monotherapy in EGFR T790M-positive NSCLC following previous EGFR-TKI therapy: CAURAL brief report. J Thorac Oncol 2019;14:933-9.

44. Yang JC, Gadgeel SM, Sequist LVD, et al. Brief report: pembrolizumab in combination with erlotinib or gefitinib as firstline therapy for advanced non-small-cell lung cancer with sensitizing EGFR mutation. J Thorac Oncol 2018;14:553-9.

45. Fulop T, Larbi A, Witkowski JM, Kotb R, Hirokawa K, Pawelec G. Immunosenescence and cancer. Crit Rev Oncog 2013;18:489-513.

46. Garassino MC, Gelibter AJ, Grossi F, et al. Italian nivolumab expanded access program in nonsquamous non-small cell lung cancer patients: results in never-smokers and EGFRmutant patients. J Thorac Oncol 2018;13:1146-55.

47. Juergens RA, Mariano C, Jolivet J, et al. Real-world benefit of nivolumab in a Canadian non-small-cell lung cancer cohort. Curr Oncol 2018;25:384-92.

48. Okuma Y, Kitano S, Watanabe K, et al. Efficacy and safety of nivolumab for cytotoxic chemotherapy unfit patients with advanced non-small cell lung cancer: a phase II study [abstract 1467P]. Ann Oncol 2018;29(suppl 8):

49. Jeremic B. Standard treatment option in stage III non-smallcell lung cancer: case against trimodal therapy and consolidation drug therapy. Clin Lung Cancer 2015;16:80-5.

50. Antonia SJ, Villegas A, Daniel D, et al. Overall survival with durvalumab after chemoradiotherapy in stage III NSCLC. N Engl J Med 2018;379:2342-50.

51. Antonia SJ, Villegas A, Daniel D, et al. Durvalumab after 
chemoradiotherapy in stage III non-small-cell lung cancer. N Engl J Med 2017;377:1919-29.

52. Chung HC, Lopez-Martin JA, Kao SCH, et al. Phase 2 study of pembrolizumab in advanced small-cell lung cancer (SCLC): KEYNOTE-158 [abstract 8506]. J Clin Oncol 2018;36:. [Available online at: https://ascopubs.org/doi/abs/10.1200/ JCO.2018.36.15_suppl.8506; cited 5 September 2019]

53. Hellmann MD, Ott PA, Zugazagoitia J, et al. Nivolumab (nivo) \pm ipilimumab (ipi) in advanced small-cell lung cancer (SCLC): first report of a randomized expansion cohort from CheckMate 032 [abstract 8503]. J Clin Oncol 2017;35:. [Available online at: https://ascopubs.org/doi/10.1200/ JCO.2017.35.15_suppl.8503; cited 5 September 2019]

54. Horn L, Mansfield AS, Szczesna A, et al. First-line atezolizumab plus chemotherapy in extensive-stage small-cell lung cancer. N Engl J Med 2018;379:2220-9. 